\title{
Chronic myeloid leukemia: cytogenetics and molecular biology's part in the comprehension and management of the pathology and treatment evolution
}

\author{
Sara Benchikh ${ }^{1,2^{*} \mathbb{D}}$, Amale Bousfiha ${ }^{2}$, Adil El Hamouchi', Somda Georgina Charlene Soro ${ }^{1,2}$, \\ Abderrahim Malki ${ }^{2}$ and Sanaa Nassereddine ${ }^{1}$
}

\begin{abstract}
Background: Chronic myelogenous leukemia (CML) is a type of blood cancer that affects hematopoietic stem cells and is often characterized by the presence of the Philadelphia chromosome. The Philadelphia chromosome encodes for a protein with high tyrosine kinase activity which acts as a tumorigenic factor.

Main body: This review article reports an update on the pathophysiology of CML and highlights the role of cytogenetic and molecular biology in screening, diagnosis, therapeutic monitoring as well as evaluating patients' response to treatment. Additionally, these genetic tests allow identifying additional chromosomal abnormalities (ACA) and BCR$\mathrm{ABL}$ tyrosine kinase domain mutations in intolerant or resistant patients. Thus, therapeutic advances have enabled this pathology to become manageable and almost curable in its clinical course. The scientific literature search used in the synthesis of this paper was carried out in the PubMed database, and the figures were generated using online software named BioRender.

Conclusion: The role of cytogenetic and molecular biology is crucial for the diagnosis and medical monitoring of patients. In-depth knowledge of molecular mechanisms of the BCR-ABL kinase facilitated the development of new targeted therapies that have improved the vital prognosis in patients. However, the emergence of ACA and new mutations resistant to tyrosine kinase inhibitors constitutes a real challenge in the quest for adequate therapy.
\end{abstract}

Keywords: CML, Tyrosine kinase, Cytogenetic, Molecular biology, Treatment resistance, Treatment response

\section{Background}

Chronic myeloid leukemia is a clonal myeloproliferative disease whose origin involves an alteration in the genome in the hematopoietic stem cell compartment. This change subsequently results in excessive production of granulocytes in the bone marrow causing both splenomegaly and hyperleukocytosis $[1,2]$.

\footnotetext{
*Correspondence: sara.benchikh1-etu@etu.univh2c.ma

${ }^{1}$ Laboratory of Cytogenetics, Pasteur Institute of Morocco, Casablanca, Morocco

Full list of author information is available at the end of the article
}

On a cytogenetic level, it is characterized by the presence of the Philadelphia chromosome, originating from a balanced reciprocal translocation $t(9 ; 22)(q 34 ; q 11)$ in more than $90 \%$ cases of CML.

This translocation causes the fusion of the Abelson $(A B L)$ proto-oncogene on chromosome 9 with the interrupted end of the breakpoint cluster region $(B C R)$ of chromosome 22 . The chimeric gene encodes a protein with high tyrosine kinase activity which acts as a tumorigenic factor $[3,4]$.

The etiological factors are globally not very known. Several epidemiological studies have demonstrated the 
effect of ionizing radiation on the development of CML in exposed people, while others suggest a possible role of exposure to benzene in the onset of the disease [5].

The median age of people affected is approximately 56 years and it accounts for about $15-20 \%$ of leukemia in adults. CML incidence worldwide varies from 0.6 to 2.8/100,000 individuals per year [6]. The age-standardized incidence rate for males was reported to be, respectively, 1.8 in Australia, 1.7 in France, 1.3 in the United States, and 0.7 in Egypt (per 100,000 individuals). For females, it was, respectively, 1 in Australia, 0.8 in France, 0.8 in the United States, and 0.8 in Egypt (per 100,000 individuals) [7].

The WHO's recent definition of CML stages divides disease progression into two phases: An accelerated phase (AP) with continuous or increasing splenomegaly, persistent or increasing leukocytosis. Blast cells count from 10 to $19 \%$, basophils count exceeds $20 \%$ and platelets count exceeds $1000 \times 10 / \mathrm{L}$. AP is also characterized by the presence of additional chromosomal aberrations (trisomy 8, isochromosome 17q, trisomy 19...) during treatment or at diagnosis as well as the occurrence of mutations (two or more) during therapy (mainly T315I). The blastic phase is characterized by $20 \%$ of blasts (in blood, bone marrow, extramedullary sites), splenomegaly and genomic evolution (emergence of ACA) [8, 9].

Cytogenetic and molecular biology techniques play a key role in the detection of chromosomal abnormalities and mutations involved in the oncogenesis of the disease. They also allow detailed cytogenetic and molecular monitoring which will contribute to the choice of the appropriate therapeutic strategy for each patient [10].

As for the treatment of CML, advances in science have led to the introduction of tyrosine kinase inhibitors, as a targeted therapy, which acts directly on the kinase activity of the BCR-ABL oncoprotein. These breakthroughs have radically changed the vital prognosis in patients with CML in terms of efficacy and survival [11].

This article aims to provide an updated review on CML and to underline the importance of cytogenetic and molecular biology in the diagnosis and therapeutic monitoring of patients, as well as to discuss the evolution of treatment in the TKI era.

\section{Main text \\ Cytogenetics of chronic myeloid leukemia}

The Philadelphia chromosome is formed as a result of a reciprocal translocation between chromosome 9 at position $q 34$ and chromosome 22 at position $q 11$. This chromosome aberration leads to the formation of a chimeric gene $B C R-A B L 1$ responsible for the oncogenic activity of the kinase (Fig. 1) [12]. According to the literature, there are several variants of simple or complex chromosomal rearrangements involved in CML (Table 2). We first mention complex translocations involving chromosomes 9 and 22 and at least one additional chromosome (from 5 to $10 \%$ ) [13]. In this case, the third chromosome is the recipient of the deleted part of $22 q$-while 9 is the recipient of the deleted part of the third chromosome. Then, simple translocations of the distal part of chromosome 22 (22ql l-qter) to another chromosome than 9. A variant characterized by a Ph chromosome which has lost its typical 22q- aspect and finally cases considered as Ph negative $(\mathrm{Ph}-)$ or atypical where chromosome 22 is intact with a breakpoint at 9q34 [14]. The most frequent variants reported in a study were the complex translocations involving chromosomes 1 and 16 [15].

\section{Chromosome 9}
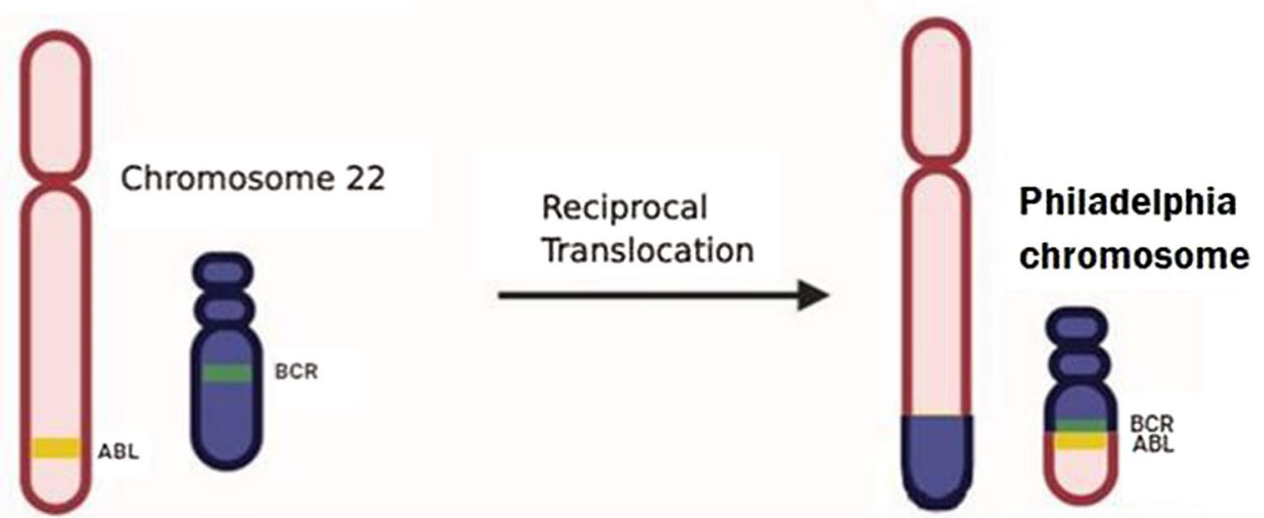

Fig. 1 Reciprocal translocation between chromosome 9 and chromosome 22 results in the formation of the Philadelphia chromosome (Created on BioRender) 
The prognostic impact of variant $\mathrm{Ph}$ translocations differs from a study to another. In one study, it was found that a variant translocation and ACA indicated a poor prognosis. Accordingly, a study suggests that the variants with four breakpoints or more confer unfavorable prognostic value [16].

While in other studies, it is suggested that variant $\mathrm{Ph}$ chromosomes have no significant effect on vital prognosis in a way that CCyR and MMR were similar in patients with or without Ph Variant $[17,18]$.

In the course of CML progression, additional chromosomal abnormalities (ACA) appear in particular during $\mathrm{AP}$ and BP and can cause genetic instability.

These abnormalities (Table 1) which are found in the cells where the Philadelphia chromosome is visible $(\mathrm{Ph}+)$ are classified into major and minor. Major pathway ACA present $10 \%$ of ACA and include mainly trisomy 8 , additional $\mathrm{Ph}$ derivation $(+\operatorname{der}(22) t(9 ; 22)$, isochromosome $17(i(17)(q 10))$, trisomy 19 and others [19]. Minor pathway ACA is less common and not sufficiently studied. It includes five aneuploidies $(-7,-17,+17,+21$, and $-\mathrm{Y})$ and one balanced structural abnormality $t(3 ; 21)(q 26$; q22) described by Mitelman [20]. In patients with $\mathrm{Ph}-$ cells, ACA found could be a reciprocal translocation $t$ (6; 9) ( $p 21 ; q 34.1)$, a chromosomal marker (+ mar), a trisomy 8 and others. On the other hand, the absence of the $\mathrm{Ph}$ chromosome and the presence of -7 (monosomy 7) contribute to the evolution towards a myelodysplastic syndrome (MDS) or acute myeloid leukemia (AML) $[12,19]$.

\section{Cytogenetic diagnosis}

In the study of hematologic malignancies, chromosome abnormalities are detected by different cytogenetic techniques. The standard karyotype aims primarily to analyze metaphases, using a light microscope, of cells blocked in mitosis [16].

When performing a karyotype, it is recommended to analyze at least 20 metaphases to assess the percentage of cells carrying the abnormality $[10,21]$. For complex karyotypes presenting additional chromosome aberrations (the presence of 3 or more chromosomal abnormalities in the same clone), the number of metaphases observed must be greater to recognize the primary and secondary abnormalities [22, 23]. In particular, a pathological karyotype indicates the presence of a Philadelphia chromosome in more than $90 \%$ of cases $(\mathrm{Ph}+)$ [14]. The $\mathrm{Ph}$ chromosome is found in all cell lines of the bone marrow: myelocytic, monocytic, megakaryoblast and erythroblast precursor cells.

The majority of B lymphocytes are $\mathrm{Ph}+$ unlike $\mathrm{T}$ lymphocytes which are $\mathrm{Ph}-$ (otherwise very rare $\mathrm{Ph}+\mathrm{T}$ cells) [12].

In molecular cytogenetics, the fluorescence in situ hybridization (FISH) technique is relatively simple and rapidly detects chromosome abnormalities. In case of masked translocation in CML suspicion, the $B C R-A B L$ fusion is sought using a complementary FISH [24]. Interphase FISH does not require cellular culture and plays an essential role in the routine analysis since some leukemic cultures do not proliferate (almost $5 \%$ of bone marrow samples) [16]. Thus, interphase FISH permits the evaluation of a large number of cells without the need for cell culture [25]. Moreover, to detect the genes involved in the pathogenesis of CML, FISH can identify all forms of the fusion gene: $t(9 ; 22)$, variant translocations, hidden translocations or insertions, using a set of two-color probes: a probe of green color to mark the $B C R$ gene, another of red color for the $A B L 1$ gene. The fusion of

Table 1 Cytogenetic variants of CML and examples of some additional chromosomal abnormalities in Ph+ cells and Ph- cells

\begin{tabular}{|c|c|}
\hline \multicolumn{2}{|l|}{ Cytogenetic variants } \\
\hline \multicolumn{2}{|c|}{ Complex translocations including chromosome 9,22 and at least one additional chromosome } \\
\hline \multicolumn{2}{|c|}{ Simple translocations of 22ql l-qter to a chromosome other than 9} \\
\hline \multicolumn{2}{|c|}{ Ph chromosome which has lost its typical 22q- aspect } \\
\hline \multicolumn{2}{|c|}{ Ph negative $(\mathrm{Ph}-)$} \\
\hline \multicolumn{2}{|c|}{ Additional chromosomal abnormalities } \\
\hline $\mathrm{Ph}+$ cells & $\mathrm{Ph}-$ cells \\
\hline Major pathway & trisomy 8 \\
\hline trisomy 8 & $t(6 ; 9)(p 21 ; q 34.1)$ \\
\hline$+\operatorname{der}(22) t(9 ; 22)$ & + mar \\
\hline \multicolumn{2}{|l|}{$i(17)(\mathrm{q} 10)$} \\
\hline \multicolumn{2}{|l|}{ trisomy 19} \\
\hline \multicolumn{2}{|l|}{ Minor pathway } \\
\hline \multicolumn{2}{|l|}{$t(3 ; 21)(\mathrm{q} 26 ; \mathrm{q} 22)$} \\
\hline$(-7,-17,+17,+21$, and $-Y)$ & \\
\hline
\end{tabular}


both probes gives a chimeric $B C R-A B L 1$ gene colored in yellow [26].

Studies indicate that, in bone marrow or peripheral blood, the FISH technique can identify less than $1 \%$ of cancer cells among 200-500 interphase nuclei [16, 27]. On the other hand, it fails to detect additional anomalies [27]. The FISH technique may also be useful in confirming complete cytogenetic remission [24].

\section{Cytogenetic response monitoring and assessment of minimal residual disease (MRD)}

The cytogenetic response is a decrease in the percentage of $\mathrm{Ph}+$ cells evaluated by analysis of residual metaphases according to conventional or molecular cytogenetics. The National Comprehensive Cancer Network (NCCN) recommends to perform the analysis at 3-6 months intervals until a complete $(\mathrm{CCyR})$ or major cytogenetic response (MCyR) is obtained [28]. CCyR is marked by the absence of $\mathrm{Ph}+$ cells which is considered as a positive prognosis for CML.

The response could be partial $(1-35 \% \mathrm{Ph}+)$, minor $(36-65 \% \mathrm{Ph}+)$ or minimal $(66-95 \% \mathrm{Ph}+)$. The loss of complete cytogenetic response is defined as the increase in $\mathrm{Ph}+$ metaphases $(\geq 30 \%)$ and serves as an indicator for therapy failure with susceptibility of disease progression $[11,29,30]$.

According to recent NCCN 2021 guidelines, cytogenetic assessment is maintained for diagnosis, failure to achieve response, loss of hematologic or cytogenetic response and thus $\mathrm{qPCR}$ has become the preferred method to monitor response to treatment [31].

\section{Treatment resistance}

TKI resistance is defined as a patient's non-response after treatment. There are two types of resistance: Primary resistance which is resistance to a medication taken for the first time and secondary resistance which is a relapse that takes place after a previous response to the treatment [29].

TKI resistance occurs through two different pathways: a BCR-ABL1 dependent or independent pathway.

Moreover, point mutations in the ABL1 domain, increase with the evolution of the pathology and become very important during the blastic phase; they can also affect biological processes leading to low TKI bioavailability [32-34].

For BCR-ABL1-dependent resistance, amplification typically through the acquisition of a second Ph chromosome or upregulation at the transcriptional level of $B C R-A B L 1$ implies the presence of additional clones and therefore a high on coprotein level, generating resistance. Moreover, point mutations of the ABL1 domain increase with disease progression mostly during blast crisis. They also can affect biological processes leading to a low TKI bioavailability [32-34].

These mutations are less detected in primary resistance than in secondary resistance to TKIs and act through various mechanisms such as (1) a change in the threedimensional conformation of the TKI-binding site, (2) stabilization of the active form of the oncoprotein so that the TKI cannot bind to its site, elevating the TKI concentrations required for inhibition above the clinically achievable range, or (3) with induction of destabilizing interactions that will abolish self-inhibition of the BCRABL1 tyrosine kinase [32, 33].

BCR-ABL1-independent pathways may be related to drug pharmacokinetics possibly influenced by CYT450 and CYP3A4 which will elevate hepatic metabolism, induce changes in the activity of efflux protein $(\mathrm{PgP})$ and impulse protein (hOCT1). The outcome would be a decrease in the intracellular concentration of the drug [35]. In addition, leukemia cells are usually characterized by genomic instability. These cancer cells can act by activating the phosphorylation of STAT3 through the cytokines of the stroma which stimulates cell growth, or by secretion of FGF2 which activates the MAPK signaling, thereby promoting leukemia cells proliferation [36]. ATP-binding cassette transporters are also involved in the development of resistance when they are overexpressed [37, 38]. Another explanation for resistance is the quiescence of LSCs, allowing these cells to persist and enter a resting phase regardless of TKI treatment. The JAK2/STAT3 and BMP4 / BMPR1B pathways are considered to be important players in signaling and promoting the persistence of residual leukemic stem cells in the bone marrow of patients [39].

A subpopulation of LSCs has been identified due to overexpression of signaling molecules such as TNF, CTNNB1, etc., considered as targets of several therapies [38]. The microenvironment is also involved in resistance via surrounding stromal cells which act on LSCs by stopping their cell cycle through growth factors and cytokines [39]. For example CXCR4, a chemokine receptor, is inhibited by the oncoprotein BCR-ABL1, causing the modification of the interactions between the medullary stroma and the LSCs [40].

TKI resistance generates TKI failure which is manifested on the cytogenetic level by the appearance of ACA. For example, the appearance of the chromosome abnormality 3q26.2 in some patients was associated with a poor vital prognosis while its presence was associated with a high frequency of the ABL1 domain mutations thus supporting TKI resistance [35]. Another study suggests that other additional major chromosomal aberrations that appear following the failure of imatinib treatment such as the addition of an extra $\mathrm{Ph}$ chromosome, or the 
isochromosome of the long arm of chromosome 17, are considered as a signal of disease progression [41].

In a randomized CML-study IV, 1510 CML patients treated with imatinib were analyzed for ACA and it was found that ACA such us $(+8,+P h, i(17 q), 3 q 26.2,-7 / 7 q$ abnormalities) also called high-risk ACA's had a negative impact on survival [42].

In another study, ACA's such as trisomy 8 , loss of $Y$ and an extra $\mathrm{Ph}$ chromosome were associated with a relatively good prognosis. In contrast, the isochromosome of the long arm of $17 i(17)(q 10)$ and 3q26.2 rearrangements were associated with a relatively poor prognosis. Furthermore, the presence of 2 or more ACA conferred an inferior survival prognosis and can be categorized into the poor prognostic group [43].

Survival results were not statistically significant or different in patients with ACA based on treatment with imatinib versus second-generation TKI following a survey [31].

\section{Molecular biology's part in the comprehension and management of CML Pathophysiology of CML}

In CML, breakpoints mainly occur in intronic regions, at the level of the $A B L 1$ and $B C R$ genes. On the $A B L 1$ gene (Fig. 2a), breakpoints are quite common between exon $1 \mathrm{~b}$ and $1 \mathrm{a}$. They can also occur upstream of exon $1 \mathrm{~b}$ or downstream of exon 1a. At the level of the $B C R$ gene (Fig. 2b), breakpoints occur mainly in the M- $B C R$ region either between exons e13 and e14, between e14 and e15, rarely in the $\mathrm{m}-B C R$ region between exon e1 and $\mathrm{e} 2$ or in the $\mathrm{u}-B C R$ region between exon e19 and e20 [44]. This recombination between $A B L 1$ and $B C R$ is responsible for the production of a variety of hybrid mRNAs (Fig. 2c). Overall, if the break takes place in the $\mathrm{M}-B C R$ region, two types of transcripts are obtained, the first e14a2 (exon 14 of $B C R$ and exon a2 of $A B L 1$ ) also called b3a2 and the second e13a2 (exon e13 $B C R$ and exon a2 of $A B L 1$ ) also called b2a2. These variants encode the typical protein of CML p210. Interruptions in the $\mathrm{m}-B C R$ or $\mathrm{u}-B C R$ regions lead, respectively, to e1a2 transcript encoding the p190 and e19a2 transcript encoding the $\mathrm{p} 230$.

P190 is mainly involved in acute lymphoblastic leukemia with Philadelphia chromosome $(\mathrm{Ph}+)$, while $\mathrm{p} 230$ is incriminated in $0.1 \%$ of chronic myeloid leukemia with polymorphonuclear cells (CML-PN) [45].

Other atypical but rare transcript variants such as e6a2, e8a2, e1a3, e13a3 and e14a3 have been reported [46].

In Table 2, studies performed in different countries have reported the frequencies of fusion transcripts among patients with $\mathrm{Ph}$ chromosome.

In the studies conducted in Indonesia, Nigeria and Iran, the b3a2 (e14a2) variant was the most commonly found in patients with respective frequencies of $74.4 \%$, $67.3 \%$ and $63 \%$. The studies established in Mexico and Sudan reveal a preponderance of the b2a2 (e13a2) with frequencies of $48 \%$ and $19.3 \%$, respectively.

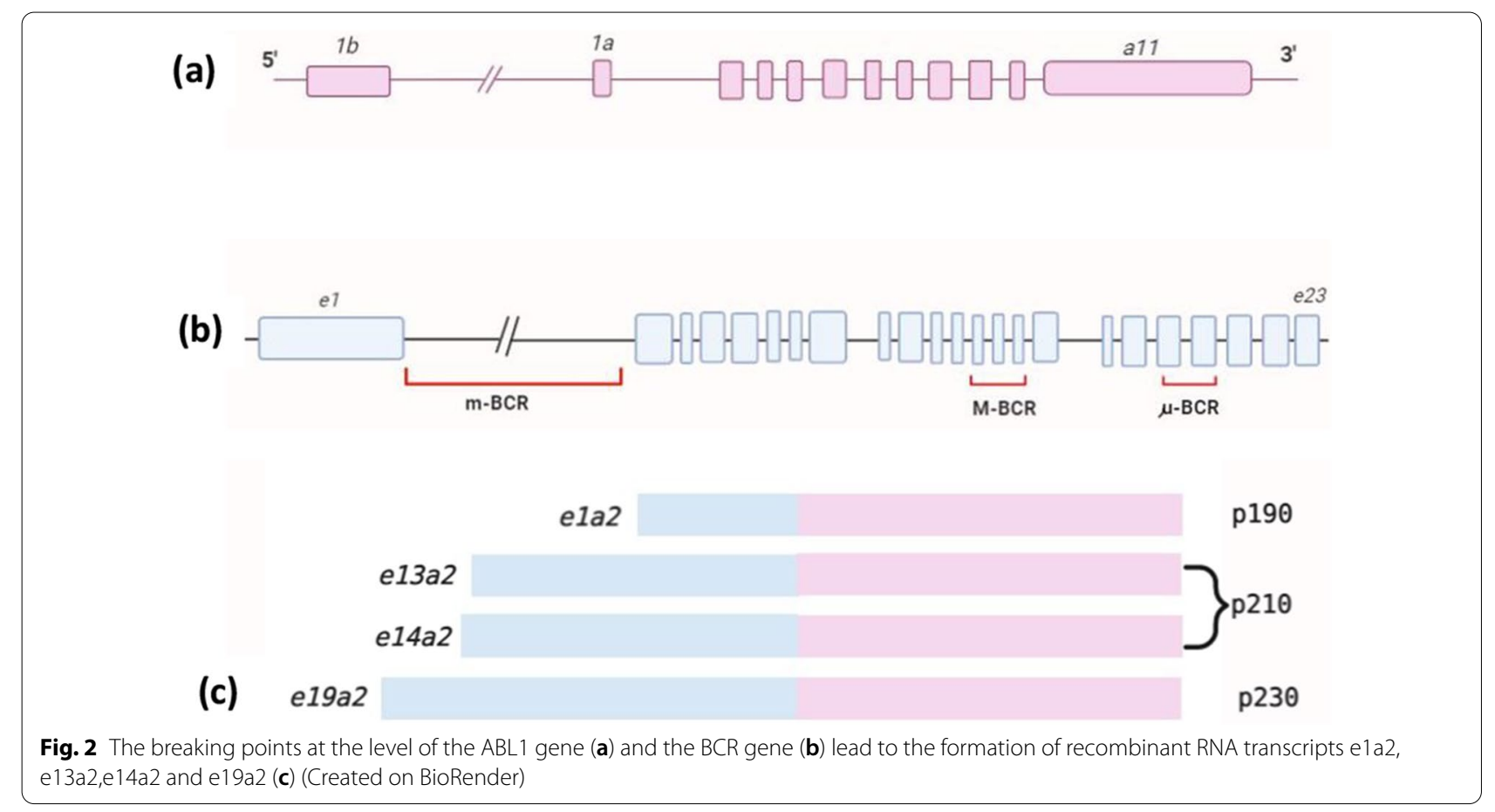


Table 2 Frequencies of expression of the $B C R-A B L$ rearrangements in patients with CML from different studies

\begin{tabular}{llllll}
\hline $\begin{array}{l}\text { Types of BCR-ABL fusion } \\
\text { transcripts }\end{array}$ & [50] Mexico $\mathbf{N = \mathbf { 2 5 0 }}$ & [51] Iran $\mathbf{N = \mathbf { 7 5 }}$ & [52] Sudan $\mathbf{N = 1 1 2}$ & [53] Nigeria $\mathbf{N = 4 2}$ & $\begin{array}{l}\text { [54] } \\
\text { Indonesia } \\
\boldsymbol{N}=\mathbf{1 8 3}\end{array}$ \\
\hline b3a2 (e14a2) & $35 \%$ & $63 \%$ & $5.5 \%$ & $67.3 \%$ & $74.3 \%$ \\
b2a2 (e13a2) & $48 \%$ & $20 \%$ & $19.3 \%$ & $21.4 \%$ & $22.4 \%$ \\
b3a2/b2a2 & $7 \%$ & - & $7.3 \%$ & - & - \\
e1a2 & - & $1 \%$ & - & - & - \\
\hline
\end{tabular}

$(N)$ : Number of patients included in the study; $(\%)$ : Frequency of the variant

The co-expression of b3a2/b2a2, was found in studies carried out in Mexico and Sudan, with an approximately equal frequency. In Indonesia, the frequency of these two co-expressed variants reaches $1 \%$.

The e1a2 variant was found in Iran with a frequency of $1 \%$.

These variations in frequencies may be linked to geographic differences or ethnic and genetic profiles.

Studies have suggested that the b3a2 and b2a2 transcripts are different in their impact on disease outcome. B3a2 appeared to have lower tyrosine kinase activity than b2a 2 and patients who expressed the b3a2 transcript treated with imatinib responded very well to treatment compared to those expressing the b2a2 transcript which appeared to have lower levels of MMR and MR4.5 [47, 48]. It has also been reported that the presence of e14a2 is associated with a high molecular response to imatinib. Overall, e14a2 transcript has better survival outcomes and patients expressing the e13a2 transcript receiving dasatinib or nilotinib responded very well to secondgeneration TKI compared to those treated with imatinib. Additionally, e1a2 and e19a2 were associated with poor cytogenetic/molecular response after TKI treatment and poor survival outcomes $[47,49]$.

The mechanism of this homologous recombination between $A B L$ and $B C R$ genes, is suggested to be related to their juxtaposition during the cell cycle (between $\mathrm{S}$ phase and G2 phase) [44].

The $\mathrm{p} 210^{\mathrm{BCR}-\mathrm{ABL}}$ oncoprotein is strongly involved in the leukemic transformation of hematopoietic stem cells thanks to the overactivation of its tyrosine kinase. It is localized in the cytoplasm and although it retains the nuclear localization sequence (NLS) and the nuclear export sequence (NES), it cannot enter the nucleus. During the blast crisis, p210 is endowed with increased activity due to the absence of negative feedback [55].

This fusion protein comprises an oligomerization domain and a tyrosine residue at position 177 which are important for the oncogenic activity of BCR-ABL1. The domain of serine-threonine kinase activity includes two binding sites to Src Homology 2 (SH2) domains, a central domain of $\mathrm{Dbl}$ homology $(\mathrm{DH})$ that functions as a guanine exchange factor (GEF), a domain of homology SRC1 (SH1) responsible for the tyrosine kinase activity, an activation loop (major autophosphorylation site), a substrate-binding site, an ATP-binding site, a SH2 domain positive regulator of the $\mathrm{SH} 1$ domain and an $\mathrm{SH} 3$ domain negative regulator of the $\mathrm{SH} 2$ domain. Lastly, a proline-rich $(P x x P)$ region that interacts with the CT10 regulator of kinase-like protein (CRKL) or Growth factor receptor-bound protein 2 (GRB2), NLS and NES sequences, a DNA binding domain and an actin-binding domain in the C-terminus of the ABL kinase (Fig. 3) [56, 57].

There are four main mechanisms involved in the induction of oncogenesis. These mechanisms are implicated in the continuous activation of the tyrosine kinase receptor (TKR) in CML: (1) gain-of-function mutations, (2) genome amplification, (3) chromosome aberrations and (4) autocrine activation by CML stem cells. BCR-ABL1 activates a variety of signaling pathways leading to cell proliferation, promotion of survival, inhibition of apoptosis, alteration of cell adhesion to the extracellular matrix and leukemic cells capability of self-renewal (Fig. 4) [57, 58].

\section{Molecular diagnosis}

Mutations are detected by qRT-PCR, a hyper-sensitive technique used to detect and quantify mRNA transcribed into complementary DNA (cDNA) which will undergo PCR amplification [59]. DNA can be labeled in a nonspecific way (without a probe) or in a specific way (with a probe). SYBR Green I is non-specific radioactive DNA marker that binds to double-stranded or single-stranded DNA. With the Taqman probe, it hybridizes to its complementary sequence (a single-stranded DNA) and in the course of polymerization, Taq polymerase degrades this probe and a fluorescent signal is released. The PCR product fluoresces as it polymerizes in both ways [60].

Some patients may express variant transcripts, which makes the diagnosis more difficult. Hence the use of the RT-multiplex PCR allows to amplify more than one target sequence using multiple primer pairs and improve the detection of Typical and atypical BCR-ABL 

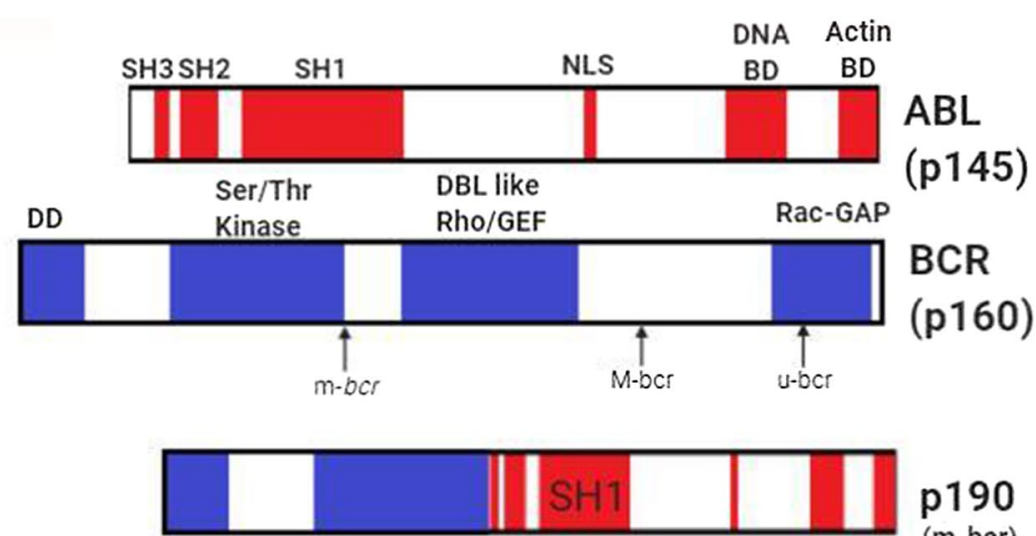

(m-bcr)

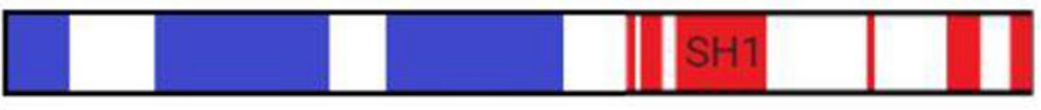

p210

(M-bcr)

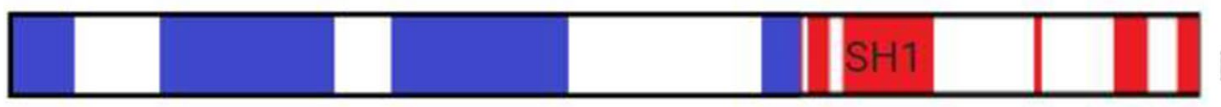

p230

(u-bcr)

Fig. 3 The resulting oncoproteins (p190, p210 and p230) of the main transcripts (Created on BioRender)

transcriptions [61]. This multiplexing can be non-specific (isoguanosine and isocytosine together) or specific by using several oligonucleotides per target [62].

\section{Molecular response monitoring and assessment of minimal residual disease (MRD)}

Molecular response is evaluated by either quantitative RT-PCR or RQ-PCR, both consisting in amplifying and quantifying the BCR-ABL fusion transcript. The RQ-PCR gives the possibility of detecting a leukemia cell out of 1 million cells allowing easy evaluation of minimal residual disease (MRD). MRD is considered to be a strong indicator of remission or relapse after treatment and an important factor in determining strategies for treatment $[29,30]$. Recently, a new method has been developed by a Japanese team, entitled in-house RQ-PCR due to its conception in their laboratory, for MRD detection and monitoring. The clinical trials revealed that this technique appears to be well standardized, very effective and sensitive in the detection of MRD and could eventually be advantageous for hospital laboratories [63].

Early molecular response (EMR) is determined by a rate of $\mathrm{BCR}-\mathrm{ABL} 1 \mathrm{IS} \leq 10 \%$ at 3 or 6 months of TKI therapy [64]. According to ELN, molecular monitoring can be classified as an optimal response post-treatment if by 3 months BCR-ABL $1 \leq 10 \%$, by 6 -months BCR$\mathrm{ABL} 1<1 \%$ and by 12 months BCR-ABL $1 \leq 0.1 \%$ marking a major molecular response (MMR), associated with a higher probability of reaching deep molecular response
(DMR) [65]. A stable molecular response is defined by persistence of the same molecular response in international scale (IS) (MMR; BCR-ABL1 IS $\leq 0.1 \%$, MR4; BCR$\mathrm{ABL} 1$ IS $\leq 0.01 \%$, and MR4.5; BCR-ABL1 IS $\leq 0.0032 \%$ ) at three consecutive assessments [66]. When the response fails post-TKI, it is defined by BCR-ABL1 $>10 \%$ at 6 -months and BCR-ABL1 $>1 \%$ at 12 -months [11].

\section{Treatment resistance}

In molecular biology, the search for mutations is carried out by conventional Sanger sequencing and more recently by next-generation sequencing (NGS) [67]. NGS allows distinguishing between polyclonal mutations that exist in different clones and compound mutations that exist within the same clone [68]. According to an investigation, the non-response to treatment in patients with CML revealed mutations undetectable by Sanger sequencing in $34 \%$ of patients knowing that these mutations are relevant to find adequate treatment [69]. Thus, NGS is used to reveal these mutations as well as their cooccurrence with somatic mutations in patients who do not respond to treatment [70].

Point mutations in the ABL1 domain are found in 50\% of patients resistant to imatinib and lead to a loss of affinity between the TKI and its binding site in the ABL1 domain [71]. More than 80 amino acid substitutions have been reported in association with resistance to imatinib. In contrast, dasatinib, nilotinib and other TKIs have a much smaller spectrum of mutations of ABL1 domain 


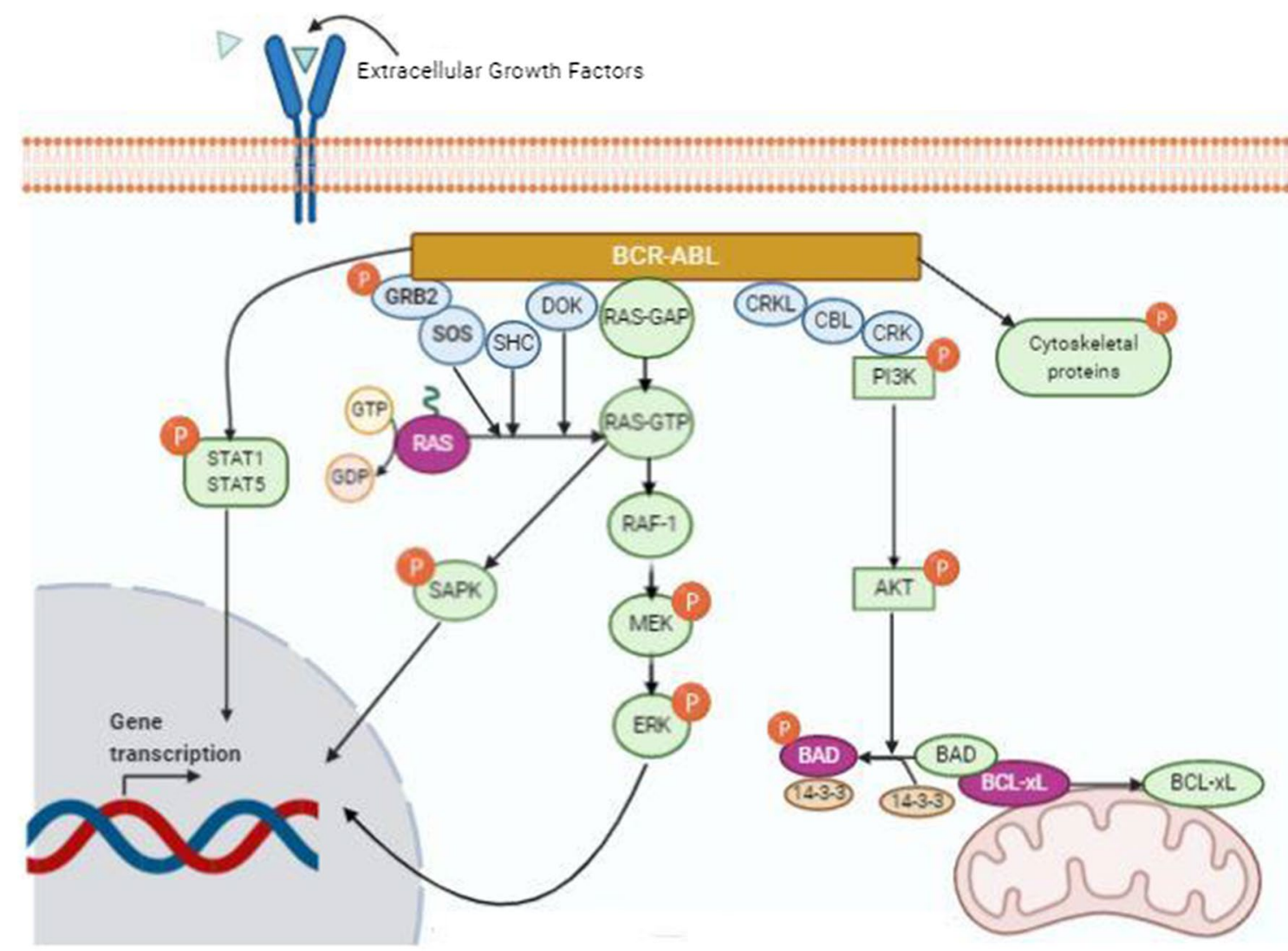

Fig. 4 Signaling pathways involved in p210 (the main protein) oncogenesis (Created on BioRender). Comment: Signalization is initiated by the TKR dimerization and activation of itself by autophosphorylation which generates the recruitment of adapter proteins [such as GRB-2, docking protein (DOK), CRKL, CRK and Casitas B-lineage Lymphoma protein (CBL)] necessary for protein to protein interactions and signal transduction. TKRs regulate several pathways and among the most important are RAS/MAPK, PI3K/AKT and JAK2/STAT

[29, 36]. Some mutations of ABL1 resistant to TKIs are cited in Fig. 5. Branford and al demonstrated that somatic variants included gene fusions, splicing abnormalities in addition to the $B C R-A B L 1$ fusion, requiring multiple modes of variant detection [72].

Recently, some other mutations have been discovered in the polycomb repressive complex $(P R C)$ pathway: PRC2 participates in gene silencing especially for the genes involved in myeloid cell line differentiation and PRC1 acts similarly on novel tumor suppressors [75].

Common mutant genes at blast crisis reported in the study of Branford were RUNX1, ASXL1 and IKZF1 exon deletions [76].

\section{Advances in treatment of chronic myeloid leukemia}

The management of chronic myeloid leukemia with $\mathrm{Ph}+$ has undergone significant progress over a relatively short period, starting with allogeneic hematopoietic stem cell transplantation and recombinant interferon alfa (rIFNa) or drugs used as initial treatment such as
Hydroxyurea, Cytarabine or Busulfan, and more recently tyrosine kinase inhibitors (TKIs) [77]. These inhibitors (Fig. 6) act directly on the kinase activity of the BCR$\mathrm{ABL}$ oncoprotein, changing radically the vital prognosis in CML patients in terms of remission and survival [78]. Approved TKIs may contribute, when combined with other antineoplastic drugs (such as interferon-a), to enhancing the efficacy of the treatment [79].

In addition, new targeted BCR-ABL1 therapies are under development, focusing primarily on third-line therapy and carriers of T315I mutation [80].

For example, Vodobatinib is a new drug studied in CML patients who are resistant/intolerant to $\geq 3$ TKIs or in the presence of comorbidities limiting the use of some TKIs. Its efficacy has been proven in vitro against most $B C R-A B L$ mutations and it is under study as a potential new agent in CML therapy [81].

Olverembatinib is a third-generation TKI proven to be effective in a phase I study in particular for patients with T315I mutation or refractory/intolerant to other TKIs [82]. 


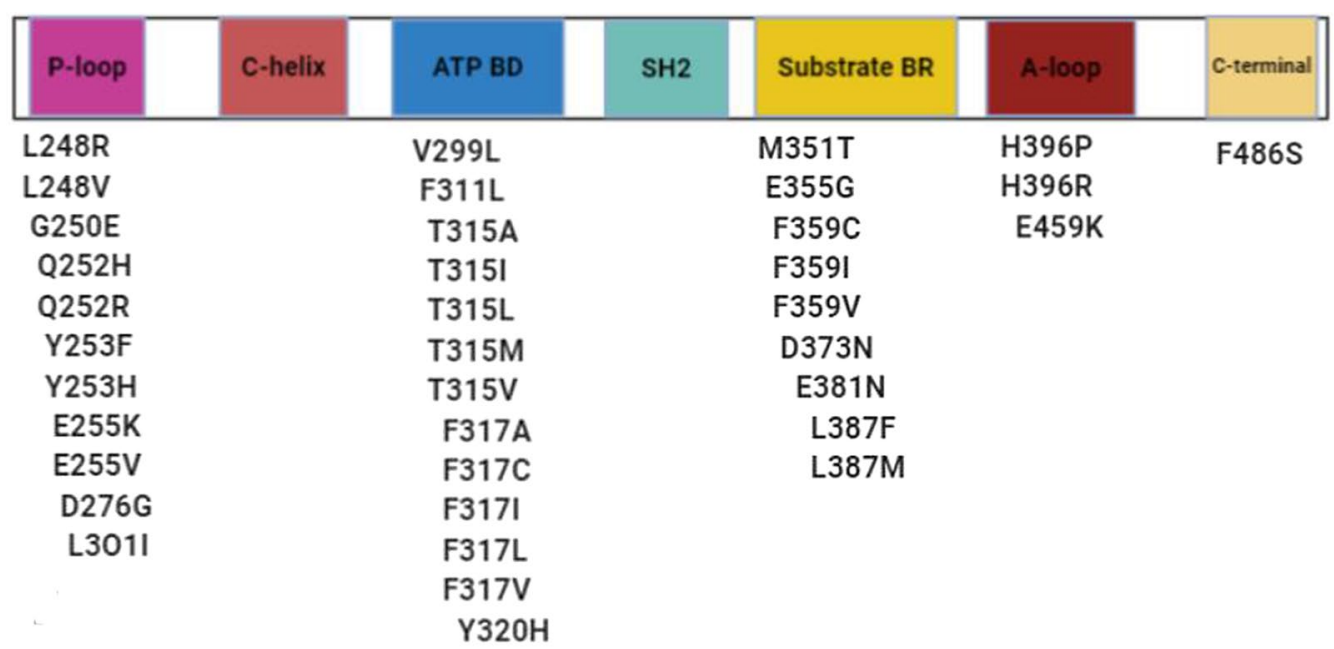

Fig. 5 Schematic representation of the kinase domain of BCR-ABL kinase showing the approximate location of resistant mutations reported in tyrosine kinase inhibitors studies. P-loop phosphate-binding loop, ATP BD ATP-binding domain, SH2 Src Homology 2 domain, Substrate BR Substrate-binding region, A-loop Activation loop [30, 33, 36, 44, 73, 74]

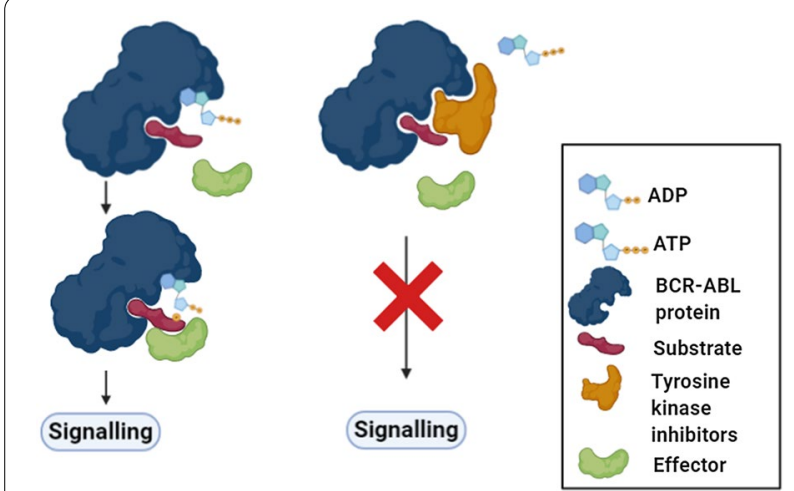

Fig. 6 ATP binding is essential for BCR-ABL to phosphorylate substrates (with a release of ADP) and then activate the signaling cascade which promotes cell survival and proliferation leading to CML. TKIs competitively bind to ATP-binding sites, thereby blocking signal transduction. Type 1 inhibitors bind to the active conformation of the kinase, while type 2 inhibitors target the inactive conformation of the kinase domain (Created on BioRender)

PF-114 is a 4th generation TKI, structurally similar to ponatinib that acts against $B C R-A B L 1$ mutations at nanomolar concentrations. This drug is effective in patients with treatment failure to 2 or more TKIs or with T315I mutation [83].

Another new targeted therapy, asciminib (ABL001), the first allosteric inhibitor of BCR-ABL1 that acts on the myristoylated site of the ABL protein and allosterically inhibits the kinase activity of the oncoprotein BCR-ABL1 by stabilizing the inactive conformation of the protein $[79,84]$.
Many clinical trials were elaborated in terms of testing the efficiency of Asciminib. Overall, they have demonstrated the effectiveness of Asciminib for patients who were resistant or intolerant to a previous type of tyrosine kinase inhibitor. In each trial, the major molecular response (MMR) was reached within a period of 24-48 weeks and the side effects noticed for the drug were approximately similar in all the studies [85-88].

Regarding therapeutic advances in hematologic malignancies, targeting non-BCR-ABL1 mediated leukemia stem cells (LSCs) offers interesting prospects to address disease persistence [89]. These leukemia stem cells are considered to be the main cause for relapse or resistance to TKIs after treatment discontinuation, because TKIs alone are unable to eradicate them [80]. The mechanism of action upon these cells aims to inhibit other molecular pathways signaling by combining TKIs with other agents to help accelerate the elimination of residual leukemia stem cells and reduce the risk for adverse events [90]. As an example of these agents: Hypomethylating agents in combination with dasatinib and BCL-2 inhibitors in combination with ponatinib which have shown encouraging results $[91,92]$.

Along with the development of treatment, treatment goals have evolved from improving the vital prognosis, preventing disease progression, minimizing treatmentrelated toxicities to treatment-free remission (TFR). Remission without treatment requires discontinuation of TKIs, which is possible in patients with prior TKI treatment for 5 years and sustained DMR for $\geq 24$ months. If these latter conditions were present, the potential to stop treatment and achieve TFR becomes possible $[66,93]$. 
In one study, DMR and duration of treatment played an important role in the success of TKI discontinuation in a group of patients [94].

Despite the possibility of treatment interruption, some patients experience molecular relapses after the loss of the MMR within the first 6 months after stopping TKIs. Recurrent patients have been reported to have fluctuating MRD measures during TFR. The rate of these patients has reached $14 \%$ after 2 years of TFR [95].

In the event of disease recurrence, a close long-term MRD monitoring is recommended to assess the molecular response in these patients, by a monthly qRT-PCR required for 6-12 months to allow rapid intervention [96].

In clinical trials for TKIS, scoring systems have been used to stratify patient risk into 3 risk groups (low, intermediate and high): the Sokal score relies on several clinical variables (number of blasts, platelets, size of the spleen and age of the patients). The Euro score relies on the same clinical variables in addition to the percentage of basophils and eosinophils in the bloodstream [97].

More recently, European Leukemia Network validated a new risk score the "European treatment and outcome study long-term survival" (ELTS) which assesses the likelihood of death from CML in patients treated with TKI, taking into account the age parameter as a negative prognostic value [98].

Another component of treatment for CML is hematopoietic stem cell transplantation (HSC) which involves transplanting these cells (from a healthy donor to a patient) to eradicate the malignant clones. Over the decades, this treatment option has been improved, deaths caused by complications after cell transplantation have decreased, and transplantation at an early age with increased safety has been allowed $[80,99]$. In the TKI era, it is considered to be a salvage therapy for patients in $\mathrm{BP}$ after responding to treatment, for patients in AP not responding to therapy or progressing towards BP phase and also for resistant/intolerant patients to multiple TKIs or at least one 2 nd generation TKIs [11, 100, 101].

\section{Conclusion}

CML is among the most studied myeloproliferative syndromes given its early discovery in the twentieth century. Following the understanding of CML's pathogenesis, scientists were able to implement new therapies for longterm remission. Cytogenetic and molecular tests, however, are crucial in the diagnosis of CML, monitoring MRD and observing the emergence of ACA or ABL1 mutations prognostically significant. In the TKI era, new drugs and drug combinations were elaborated to overcome resistance, intolerance and eliminate residual LSCs. For patients refractory to TKI in the advanced phase of the disease, allogeneic transplantation remains the best alternative solution. In general, chronic myeloid leukemia has become manageable and curable over the decades thanks to advances in cancer therapy. However, achieving remission without treatment is still controversial and remains a challenge after treatment discontinuation.

\begin{abstract}
Abbreviations
CML: Chronic myeloid leukemia; ACA: Additional chromosomal abnormalities; $B C R$ : Breakpoint cluster region; ABL: Abelson; TKI: Tyrosine kinase inhibitors; WHO: World health organization; AP: Accelerated phase; Ph+: Philadelphia chromosome; CCyR: Complete cytogenetic response; MMR: Major molecular response; MDS: Myelodysplastic syndrome; AML: Acute myeloid leukemia; FISH: Fluorescence in situ hybridization; MRD: Minimal residual disease; NCCN: National comprehensive cancer network; MCyR: Major cytogenetic response; PCR: Polymerase chain reaction; CYT450: Cytochrome P450; CYP3A4: Cytochrome P450 3A4; PgP: P-glycoprotein 1; hOCT1: Human organic cation transporter 1; STAT3: Signal transducer and activator of transcription 3; FGF2: Fibroblast growth factor 2; MAPK: Mitogen-activated protein kinases; ATP: Adenosine triphosphate; LSCs: Leukemia stem cells; JAK2: Janus kinase 2; BMP4: Bone morphogenetic protein 4; BMPR: Bone morphogenetic protein receptor; TNF: Tumor necrosis factor; CTNNB1: Catenin beta 1; CXCR4: C-X-C motif chemokine receptor 4; RNA: Ribonucleic acid; NLS: Nuclear localization sequence; NES: Nuclear export sequence; SH2: Src homology 2; DH: Dbl homology; GEF: Guanine exchange factor; SH1: SRC homology 1; Pxxp: Proline-rich region; CRKL: CT10 regulator of kinase-like protein; GRB2: Growth factor receptor-bound protein 2; DNA: Deoxyribonucleic acid; qRT-PCR: Quantitative reverse transcriptase polymerase chain reaction; EMR: Early molecular response; DMR: Deep molecular response; NGS: Next generation sequencing; PRC: Polycomb repressive complex; RUNX1: Runt-related transcription factor 1; ASXL1: ASXL transcriptional regulator 1; IKZF1: Ikaros zinc finger protein 1; IFN: Interferon; TFR: Treatment free remission; ELTS: European treatment and outcome study long term survival score; HSC: Hematopoietic stem cells; BP: Blastic phase; ADP: Adenosine diphosphate.
\end{abstract}

\section{Acknowledgements}

Not applicable.

\section{Authors' contributions}

$S B$ was a major contributor in writing the manuscript. Coauthors $A B, A H$, $\mathrm{SN}, \mathrm{AM}$ and SS gave critical analysis for the manuscript. All authors read and approved the final manuscript.

\section{Funding}

No funds, grants, or other support was received.

Availability of data and materials

Not applicable.

\section{Declarations}

Ethics approval and consent to participate

This article does not contain any studies with human or animal subjects performed by any of the authors.

\section{Consent for publication}

Not applicable.

\section{Competing interests}

The authors have no conflict of interest to declare that are relevant to the content of this article.

\section{Author details}

${ }^{1}$ Laboratory of Cytogenetics, Pasteur Institute of Morocco, Casablanca, Morocco. ${ }^{2}$ Laboratory of Physiopathology and Molecular Genetics, Faculty of Sciences Ben M'Sik, Hassan II University, Casablanca, Morocco. 
Received: 29 June 2021 Accepted: 2 January 2022

Published online: 06 March 2022

\section{References}

1. Nowell P, Hungerford D (1960) A minute chromosome in human chronic granulocytic leukemia. ScienceOpen, Berlin

2. Rowley JD (1973) Letter: A new consistent chromosomal abnormality in chronic myelogenous leukaemia identified by quinacrine fluorescence and Giemsa staining. Nature 243(5405):290-293

3. Heisterkamp N, Stephenson JR, Groffen J, Hansen PF, de Klein A, Bartram CR et al (1983) Localization of the c-ab1 oncogene adjacent to a translocation break point in chronic myelocytic leukaemia. Nature 306(5940):239-242

4. Groffen J, Stephenson JR, Heisterkamp N, de Klein A, Bartram CR, Grosveld G (1984) Philadelphia chromosomal breakpoints are clustered within a limited region, bcr, on chromosome 22. Cell 36(1):93-99

5. Vlaanderen J, Lan Q, Kromhout H, Rothman N, Vermeulen R (2012) Occupational benzene exposure and the risk of chronic myeloid leukemia: a meta-analysis of cohort studies incorporating study quality dimensions. Am J Ind Med 55(9):779-785

6. Rohrbacher M, Hasford J (2018) Epidemiology and etiology of chronic myeloid leukemia. In: Wiernik PH, Dutcher JP, Gertz MA (eds) Neoplastic diseases of the blood. Springer, Cham, pp 9-17

7. Miranda-Filho A, Piñeros M, Ferlay J, Soerjomataram I, Monnereau A, Bray F (2018) Epidemiological patterns of leukaemia in 184 countries: a population-based study. Lancet Haematol 5(1):e14-24

8. Hochhaus A, Saussele S, Rosti G, Mahon F-X, Janssen JJWM, HjorthHansen $\mathrm{H}$ et al (2017) Chronic myeloid leukaemia: ESMO clinica practice guidelines for diagnosis, treatment and follow-up. Ann Oncol 28:41-51

9. Haznedaroğlu iC, Kuzu I, IIlhan O (2020) WHO 2016 definition of chronic myeloid leukemia and tyrosine kinase inhibitors. Turk J Hematol 37(1):42-47

10. Dorfman LE, Floriani MA, Oliveira TMRDR, Cunegatto B, Rosa RFM, Zen PRG (2018) The role of cytogenetics and molecular biology in the diagnosis, treatment and monitoring of patients with chronic myeloid leukemia. J Bras Patol E Med Lab 54(2):83-91

11. Hochhaus A, Baccarani M, Silver RT, Schiffer C, Apperley JF, Cervantes F et al (2020) European LeukemiaNet 2020 recommendations for treating chronic myeloid leukemia. Leukemia 34(4):966-984

12. Asnafi AA, Deris Zayeri Z, Shahrabi S, Zibara K, Vosughi T (2019) Chronic myeloid leukemia with complex karyotypes: prognosis and therapeutic approaches. J Cell Physiol 234(5):5798-5806

13. Rohan B, Purohit S, Lokanatha D, Jacob L, Suresh TM, Govind Babu K et al (2012) Chronic myeloid leukemia with variant chromosomal translocations: results of treatment with imatinib mesylate. J Health Allied Sci 11:1-4

14. Hagemeijer A (1987) Chromosome abnormalities in CML. Baillières Clin Haematol 1(4):963-981

15. Shetty D, Talker E, Jain H, Talker J, Patkar N, Subramanian P et al (2021) Evaluation of cytogenetic response in CML patients with variant Philadelphia translocation. Asia Pac J Clin Oncol. https://doi.org/10.1111/ ajco.13522

16. Ankathil R, Ismail SM, Mohd Yunus N, Sulong S, Husin A, Abdullah AD et al (2020) Clinical implications of conventional cytogenetics, fluorescence in situ hybridization (FISH) and molecular testing in chronic myeloid leukaemia patients in the tyrosine kinase inhibitor era-a review. Malays J Pathol 42(3):307-321

17. Eyüpoğlu D, Bozkurt S, Haznedaroğlu İ, Büyükaşık Y, Güven D (2016) The impact of variant Philadelphia chromosome translocations on the clinical course of chronic myeloid leukemia. Turk J Hematol 33(1):60-65

18. Trivedi P, Varma P, Patel D, Ladani D, Patel D, Kazi M et al (2019) Clinica implications of simultaneous occurrence of variant Philadelphia translocations in chronic myeloid leukemia. J Assoc Genet Technol 45(2):61-65

19. Sgherza N, Abruzzese E, Perla G, Minervini MM, Chiello V, Sciannamè N et al (2017) Onset of chronic myeloid leukemia with complex karyotype in a pregnant patient: case report and revision of literature. Ther Clin Risk Manag 27(13):751-755
20. Mitelman F (1993) The cytogenetic scenario of chronic myeloid leukemia. Leuk Lymphoma 11(Suppl 1):11-15

21. Benchikh S, Bousfiha A, Razoki L, Aboulfaraj J, Zarouf L, Elbakay C et al (2021) Chromosome abnormalities related to reproductive and sexual development disorders: a 5-year retrospective study. BioMed Res Int 5(2021):1-11

22. Fröhling S, Döhner H (2008) Chromosomal abnormalities in cancer. N Engl J Med 359(7):722-734

23. Willatt L, Morgan S (2009) ISCN 2009 an international system for human cytogenetic nomenclature. Hum Genet 126:603-604

24. Roche-Lestienne C, Boudry-Labis E, Mozziconacci M-J (2016) Cytogenetics in the management of "chronic myeloid leukemia": an update by the Groupe francophone de cytogénétique hématologique (GFCH). Ann Biol Clin (Paris) 74(5):511-515

25. Huegel A, Coyle L, McNeil R, Smith A (1995) Evaluation of interphase fluorescence in situ hybridization on direct hematological bone marrow smears. Pathology (Phila) 27(1):86-90

26. Levsky JM, Singer RH (2003) Fluorescence in situ hybridization: past, present and future. J Cell Sci 116(Pt 14):2833-2838

27. Wolff DJ, Bagg A, Cooley LD, Dewald GW, Hirsch BA, Jacky PB et al (2007) Guidance for fluorescence in situ hybridization testing in hematologic disorders. J Mol Diagn 9(2):134-143

28. Radich JP, Deininger M, Abboud CN, Altman JK, Berman E, Bhatia R et al (2018) Chronic myeloid leukemia, version 1.2019, NCCN clinical practice guidelines in oncology. J Natl Compr Cancer Netw JNCCN 16(9):1108-1135

29. Baccarani M, Deininger MW, Rosti G, Hochhaus A, Soverini S, Apperley JF et al (2013) European LeukemiaNet recommendations for the management of chronic myeloid leukemia. Blood 122(6):872-884

30. Steegmann JL, Baccarani M, Breccia M, Casado LF, García-Gutiérrez V, Hochhaus A et al (2016) European LeukemiaNet recommendations for the management and avoidance of adverse events of treatment in chronic myeloid leukaemia. Leukemia 30(8):1648-1671

31. Deininger MW, Shah NP, Altman JK, Berman E, Bhatia R, Bhatnagar B et al (2020) Chronic myeloid leukemia, version 2.2021, NCCN clinical practice guidelines in oncology. J Natl Compr Cancer Netw 18(10):1385-1415

32. Nestal de Moraes G, Souza PS, Costas FCF, Vasconcelos FC, Reis FRS, Maia RC (2012) The interface between BCR-ABL-dependent and -independent resistance signaling pathways in chronic myeloid leukemia. Leuk Res Treat 2012:19

33. Patel $A B, O^{\prime}$ Hare T, Deininger MW (2017) Mechanisms of resistance to $A B L$ kinase inhibition in chronic myeloid leukemia and the development of next generation ABL kinase inhibitors. Hematol Oncol Clin North Am 31(4):589-612

34. Zhao H, Deininger MW (2020) Declaration of Bcr-Abl1 independence. Leukemia 34(11):2827-2836

35. Zhou T, Medeiros LJ, Hu S (2018) Chronic myeloid leukemia: beyond BCR-ABL1. Curr Hematol Malig Rep 13(6):435-445

36. Braun TP, Eide CA, Druker BJ (2020) Response and resistance to BCRABL1-targeted therapies. Cancer Cell 37(4):530-542

37. Ding Y, Fan J, Fan Z, Zhang K (2021) $Y$-Tocotrienol reverses multidrug resistance of breast cancer cells through the regulation of the Y-Tocotrienol-NF-kB-P-gp axis. J Steroid Biochem Mol Biol 209:105835

38. Loscocco F, Visani G, Galimberti S, Curti A, Isidori A (2019) BCR-ABL independent mechanisms of resistance in chronic myeloid leukemia. Front Oncol 9:939

39. Jeanpierre S, Arizkane K, Thongjuea S, Grockowiak E, Geistlich K, Barral $L$ et al (2021) The quiescent fraction of chronic myeloid leukemic stem cells depends on BMPR1B, Stat3 and BMP4-niche signals to persist in patients in remission. Haematologica 106(1):111-122

40. O'Reilly E, Zeinabad HA, Nolan C, Sefy J, Williams T, Tarunina M et al (2021) Recreating the bone marrow microenvironment to model leukemic stem cell quiescence. Front Cell Dev Biol 9:2319

41. Villatoro A, Konieczny J, Cuminetti V, Arranz L (2020) Leukemia stem cell release from the stem cell niche to treat acute myeloid leukemia. Front Cell Dev Biol 8:607

42. Meggyesi N, Kozma A, Halm G, Nahajevszky S, Bátai Á, Fekete S et al (2012) Additional chromosome abnormalities, BCR-ABL tyrosine kinase domain mutations and clinical outcome in Hungarian tyrosine kinase 
inhibitor-resistant chronic myelogenous leukemia patients. Acta Haematol 127(1):34-42

43. Hehlmann R, Voskanyan A, Lauseker M, Pfirrmann M, Kalmanti L, Rinaldetti S et al (2020) High-risk additional chromosomal abnormalities at low blast counts herald death by CML. Leukemia 34(8):2074-2086

44. Wang W, Cortes JE, Tang G, Khoury JD, Wang S, Bueso-Ramos CE et al (2016) Risk stratification of chromosomal abnormalities in chronic myelogenous leukemia in the era of tyrosine kinase inhibitor therapy. Blood 127(22):2742-2750

45. Quintás-Cardama A, Cortes J (2009) Molecular biology of bcr-abl1-positive chronic myeloid leukemia. Blood 113(8):1619-1630

46. Leguay T, Mahon F-X (2005) Leucémie myéloïde chronique. EMC Hématol 2(3):187-205

47. Burmeister T, Reinhardt R (2008) A multiplex PCR for improved detection of typical and atypical BCR-ABL fusion transcripts. Leuk Res 32(4):579-585

48. Genthon A, Nicolini FE, Huguet F, Colin-Gil C, Berger M, Saugues S et al (2020) Influence of major BCR-ABL1 transcript subtype on outcome in patients with chronic myeloid leukemia in chronic phase treated frontline with nilotinib. Oncotarget 11(26):2560-2570

49. Nachi M, Kihel I, Entasoltane B, Brahimi M, Yafour N, Guella D et al (2020) Impact of the major BCR-ABL1 transcript type on clinical and biological parameters and molecular response in patients with chronic myeloid leukemia. Hematol Oncol Stem Cell Ther. https://doi.org/10.1016/j. hemonc.2020.08.003

50. Marcé S, Xicoy B, García O, Cabezón M, Estrada N, Vélez P et al (2021) Impact of BCR-ABL1 transcript type on response, treatment-free remission rate and survival in chronic myeloid leukemia patients treated with imatinib. J Clin Med 10(14):3146

51. Arana-Trejo RM, Sánchez ER, Ignacio-Ibarra G, De La Fuente EB, Garces O, Morales EG et al (2002) BCR/ABL p210, p190 and p230 fusion genes in 250 Mexican patients with chronic myeloid leukaemia (CML): BCR/ABL in CML Mexican patients. Clin Lab Haematol 24(3):145-150

52. Yaghmaie M, Ghaffari SH, Ghavamzadeh A, Alimoghaddam K, Jahani M, Mousavi S-A, et al. Frequency of BCR-ABL fusion transcripts in iranian patients with chronic myeloid leukemia. 2008;5.

53. Muddathir AR, Kordofani A, Fadl-Elmula I (2013) Frequency of BCR-ABL fusion transcripts in Sudanese patients with chronic myeloid leukemia using real-time reverse transcription-polymerase chain reaction. Saudi Med J 9(34):29-33

54. Uzoma IC, Taiwo IA, Nna EO, Durosinmi MA, Ukaejiofo EO (2019) Detection of BCR-ABL1 fusion gene transcripts in the saliva of Nigerian patients with chronic myeloid leukemia. Niger J Clin Pract 22(1):51-55

55. Paramita DK, Hutajulu SH, Syifarahmah A, Sholika TA, Fatmawati S, Aning S et al (2020) BCR-ABL gene transcript types of patients with chronic myelogenous leukemia in Yogyakarta, Indonesia. Asian Pac J Cancer Prev APJCP 21(6):1545-1550

56. Perrotti D, Calabretta B (2004) Translational regulation by the p210 BCR/ ABL oncoprotein. Oncogene 23(18):3222-3229

57. Chalandon Y, Jiang X, Hazlewood G, Loutet S, Conneally E, Eaves A et al (2002) Modulation of p210BCR-ABL activity in transduced primary human hematopoietic cells controls lineage programming. Blood 99(9):3197-3204

58. Goldman JM, Melo JV (2003) Chronic myeloid leukemia_advances in biology and new approaches to treatment. N Engl J Med 349(15):1451-1464

59. Du Z, Lovly CM (2018) Mechanisms of receptor tyrosine kinase activation in cancer. Mol Cancer 17(1):58

60. Farkas DH, Holland CA (2009) Overview of molecular diagnostic techniques and instrumentation. Cell and tissue based molecular pathology. Elsevier, pp 19-32

61. Bustin SA, Mueller R (2005) Real-time reverse transcription PCR (qRTPCR) and its potential use in clinical diagnosis. Clin Sci 109(4):365-379

62. Mir R, Ahmad I, Javid J, Zuberi M, Yadav P, Shazia R et al (2015) Simple multiplex RT-PCR for identifying common fusion BCR-ABL transcript types and evaluation of molecular response of the $\mathrm{a} 2 \mathrm{~b} 2$ and $\mathrm{a} 2 \mathrm{~b} 3 \mathrm{tran}$ scripts to Imatinib resistance in north Indian chronic myeloid leukemia patients. Indian J Cancer 52(3):314-318

63. Limsuwanachot N, Siriboonpiputtana T, Karntisawiwat K, Chareonsirisuthigul T, Chuncharunee S, Rerkamnuaychoke B (2016) Multiplex RT-PCR Assay for detection of common fusion transcripts in acute lymphoblastic leukemia and chronic myeloid leukemia cases. Asian Pac J Cancer Prev APJCP 17(2):677-684

64. Kitamura H, Tabe Y, Ai T, Tsuchiya K, Yuri M, Misawa S et al (2019) A new highly sensitive real-time quantitative-PCR method for detection of BCR-ABL1 to monitor minimal residual disease in chronic myeloid leukemia after discontinuation of imatinib. Jiang $X$, éditeur. PLOS ONE 14(3):170

65. Ono T (2021) Which tyrosine kinase inhibitors should be selected as the first-line treatment for chronic myelogenous leukemia in chronic phase? Cancers 13(20):5116

66. Shanmuganathan N, Hughes TP (2018) Molecular monitoring in CML: how deep? How often? How should it influence therapy? Hematol Am Soc Hematol Educ Program 2018(1):168-176

67. Branford S (2020) Why is it critical to achieve a deep molecular response in chronic myeloid leukemia? Haematologica 105(12):2730-2737

68. Miura T, Yasuda S, Sato Y (2021) A simple method to estimate the in-house limit of detection for genetic mutations with low allele frequencies in whole-exome sequencing analysis by next-generation sequencing. BMC Genom Data 22(1):8

69. Soverini S, Abruzzese E, Bocchia M, Bonifacio M, Galimberti S, Gozzini A et al (2019) Next-generation sequencing for BCR-ABL1 kinase domain mutation testing in patients with chronic myeloid leukemia: a position paper. J Hematol OncolJ Hematol Oncol 12:131

70. Soverini S, Bavaro L, De Benedittis C, Martelli M, lurlo A, Orofino N et al (2020) Prospective assessment of NGS-detectable mutations in CML patients with nonoptimal response: the NEXT-in-CML study. Blood 135(8):534-541

71. Curik N, Polivkova V, Burda P, Koblihova J, Laznicka A, Kalina T et al (2021) Somatic mutations in oncogenes are in chronic myeloid leukemia acquired de novo via deregulated base-excision repair and alternative non-homologous end joining. Front Oncol 11:3795

72. Soverini S, Abruzzese E, Bocchia M, Bonifacio M, Galimberti S, Gozzini A et al (2019) Next-generation sequencing for BCR-ABL1 kinase domain mutation testing in patients with chronic myeloid leukemia: a position paper. J Hematol Oncol J Hematol Oncol 12(1):131

73. Branford S, Wang P, Yeung DT, Thomson D, Purins A, Wadham C et al (2018) Integrative genomic analysis reveals cancer-associated mutations at diagnosis of CML in patients with high-risk disease. Blood 132(9):948-961

74. Shah NP, Nicoll JM, Nagar B, Gorre ME, Paquette RL, Kuriyan J et al (2002) Multiple BCR-ABL kinase domain mutations confer polyclonal resistance to the tyrosine kinase inhibitor imatinib (ST1571) in chronic phase and blast crisis chronic myeloid leukemia. Cancer Cell 2(2):117-125

75. Chandrasekhar C, Kumar PS, Sarma PVGK (2019) Novel mutations in the kinase domain of BCR-ABL gene causing imatinib resistance in chronic myeloid leukemia patients. Sci Rep 9(1):2412

76. Ko TK, Javed A, Lee KL, Pathiraja TN, Liu X, Malik S et al (2020) An integrative model of pathway convergence in genetically heterogeneous blast crisis chronic myeloid leukemia. Blood 135(26):2337-2353

77. Branford S, Kim DDH, Apperley JF, Eide CA, Mustjoki S, Ong ST et al (2019) Laying the foundation for genomically-based risk assessment in chronic myeloid leukemia. Leukemia 33(8):1835-1850

78. Goldman JM (2010) Chronic myeloid leukemia: a historical perspective. Semin Hematol 47(4):302-311

79. Sharf G, Marin C, Bradley JA, Pemberton-Whiteley Z, Bombaci F, Christensen RIO et al (2020) Treatment-free remission in chronic myeloid leukemia: the patient perspective and areas of unmet needs. Leukemia 34(8):2102-2112

80. Westerweel PE, Te Boekhorst PAW, Levin M-D, Cornelissen JJ (2019) New approaches and treatment combinations for the management of chronic myeloid leukemia. Front Oncol 9:665

81. Cortes J, Lang F (2021) Third-line therapy for chronic myeloid leukemia: current status and future directions. J Hematol Oncol J Hematol Oncol 14(1):44

82. Cortes J. Phase 1 Trial of Vodobatinib, a Novel Oral BCR-ABL1 tyrosine kinase inhibitor (TKI): activity in CML chronic phase patients failing TKI therapies including ponatinib. In ASH; 2020.

83. Lu M. Exposure-response (E-R) analysis of olverembatinib (HQP1351) in Chinese patients with chronic myeloid leukemia (CML). In: ASH; 2020.

84. Turkina A, Vinogradova O, Lomaia E, Shatokhina E, Shukhov O, Chelysheva E et al (2019) PF-114: a 4th generation tyrosine kinase-inhibitor 
for chronic phase chronic myeloid leukaemia including BCRABL1T315I. Blood 13(134):1638-1638

85. Eide CA, Zabriskie MS, Savage Stevens SL, Antelope O, Vellore NA Than $\mathrm{H}$ et al (2019) Combining the allosteric inhibitor asciminib with ponatinib suppresses emergence of and restores efficacy against highly resistant BCR-ABL1 mutants. Cancer Cell 36(4):431-443

86. Cortes J, Lang F, Kim D-W, Réa D, Mauro MJ, Minami H et al (2019) S883 combination therapy using asciminib plus imatinib (IMA) in patients (PTS) with chronic myeloid leukemia (CML): results from a phase 1 study. HemaSphere 3(S1):397

87. Hochhaus A, Boquimpani C, Rea D, Minami Y, Lomaia E, Voloshin S et al (2020) Efficacy and safety results from ASCEMBL, a multicenter, open-label, phase 3 study of asciminib, a first-in-class STAMP inhibitor, vs bosutinib (BOS) in patients (Pts) with chronic myeloid leukemia in chronic phase (CML-CP) previously treated with $\geq 2$ tyrosine kinase inhibitors (TKIs). Blood 136:LBA4

88. Hughes T, Mauro M, Cortes J, Minami H, Rea D, DeAngelo D et al (2019) Asciminib in chronic myeloid leukemia after ABL kinase inhibitor failure. N Engl J Med 12(381):2315-2326

89. Hughes $T$ et al (2020) CML-121: efficacy and safety of asciminib in heavily pretreated patients with Philadelphia chromosome-positive $(\mathrm{Ph}+)$ chronic myeloid leukemia in chronic phase (CML-CP) with sensitivity to tyrosine kinase inhibitors (TKIs). Clin Lymphoma Myeloma Leukemia 20:S235

90. Muselli F, Peyron J-F, Mary D (2019) Druggable biochemical pathways and potential therapeutic alternatives to target leukemic stem cells and eliminate the residual disease in chronic myeloid leukemia. Int J Mol Sci 20(22):5616

91. Özgür Yurttaş N, Eşkazan AE (2020) Novel therapeutic approaches in chronic myeloid leukemia. Leuk Res 91:106337

92. Abaza Y, Kantarjian H, Alwash Y, Borthakur G, Champlin R, Kadia T et al (2020) Phase I/II study of dasatinib in combination with decitabine in patients with accelerated or blast phase chronic myeloid leukemia. Am J Hematol 95(11):1288-1295

93. Maiti A, Franquiz MJ, Ravandi F, Cortes JE, Jabbour EJ, Sasaki Ket al (2020) Venetoclax and BCR-ABL tyrosine kinase inhibitor combinations: outcome in patients with philadelphia chromosome-positive advanced myeloid leukemias. Acta Haematol 143(6):567-573

94. Atallah E, Schiffer CA (2020) Discontinuation of tyrosine kinase inhibitors in chronic myeloid leukemia: When and for whom? Haematologica 105(12):2738-2745

95. Chen K, Du T, Xiong P, Fan G, Yang W (2019) Discontinuation of tyrosine kinase inhibitors in chronic myeloid leukemia with losing major molecular response as a definition for molecular relapse: a systematic review and meta-analysis. Front Oncol 9:372

96. Rousselot P, Loiseau C, Delord M, Cayuela JM, Spentchian M (2020) Late molecular recurrences in patients with chronic myeloid leukemia experiencing treatment-free remission. Blood Adv 4(13):3034-3040

97. Merli P, Ifversen M, Truong TH, Marquart HV, Buechner J, Wölfl M et al (2021) Minimal residual disease prior to and after haematopoietic stem cell transplantation in children and adolescents with acute lymphoblastic leukaemia: What level of negativity is relevant? Front Pediatr 9:1279

98. Aijaz J, Junaid N, Asif Naveed M, Maab R (2020) Risk stratification of chronic myeloid leukemia according to different prognostic scores. Cureus 12(3):e7342

99. Pfirrmann M, Clark RE, Prejzner W, Lauseker M, Baccarani M, Saussele S et al (2020) The EUTOS long-term survival (ELTS) score is superior to the Sokal score for predicting survival in chronic myeloid leukemia. Leukemia 34(8):2138-2149

100. Taylor M, Khan S, Stapleton M, Wang J, Chen J, Wynn R et al (2019) Hematopoietic stem cell transplantation for mucopolysaccharidoses; past, present, and future. Biol Blood Marrow Transplant J Am Soc Blood Marrow Transplant 25(7):e226

101. Jabbour E, Kantarjian H (2020) Chronic myeloid leukemia: 2020 update on diagnosis, therapy and monitoring. Am J Hematol 95(6):691-709

\section{Publisher's Note}

Springer Nature remains neutral with regard to jurisdictional claims in published maps and institutional affiliations.

\section{Submit your manuscript to a SpringerOpen ${ }^{\circ}$ journal and benefit from:}

- Convenient online submission

- Rigorous peer review

- Open access: articles freely available online

- High visibility within the field

- Retaining the copyright to your article

Submit your next manuscript at $\boldsymbol{\nabla}$ springeropen.com 